\title{
Osteoarthritis Research Society International is a Pioneer in Recommendations for Histological Evaluation in Basic Research
}

\author{
Fernando Augusto Vasilceac* and Camila Marques de Araújo
}

Departament of Gerontology, Federal University of Sao Carlos, Brazil

ISSN: 2578-0093

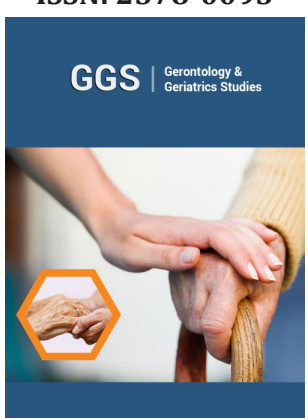

*Corresponding author: Fernando Augusto Vasilceac, Department of Gerontology, Brazil

Submission: 韭April 07, 2020

Published: 陋April 20, 2020

Volume 5 - Issue 5

How to cite this article: Fernando Augusto Vasilceac, Camila Marques de Araújo. Osteoarthritis Research Society International is a Pioneer in Recommendations for Histological Evaluation in Basic Research. Gerontol \& Geriatric stud. 5(5). GGS.000623. 2020. DOI: $10.31031 /$ GGS.2020.05.000623

Copyright@ Fernando Augusto Vasilceac, This article is distributed under the terms of the Creative Commons Attribution 4.0 International License, which permits unrestricted use and redistribution provided that the original author and source are credited.

\section{Opinion}

Basic research in the area of indirect research promotes public politics and preventive programs to help health care, using as a main tool the thorough investigation of pathological processes of different clinical conditions. In osteoarthritis, the rates of medical appointments and leave from work can be minimized with an understanding of all the mechanisms involved in the disease. An osteoarthritis presents a sequence of morphological changes characterized by the loss of the normal configuration of the extracellular cartilage matrix, crackling on movement, bone deformities, formation of osteophytes and increased synovial fluid. Pain is not the first clinical sign of the disease, mainly due to the a neural and avascular resources of the articular cartilage, but it is consensual in the literature that since the beginning of the disease existing in the inflammatory process in the joints [1].

Proinflammatory cytokines such as interleukin-1 beta (IL-1 $\beta$ ) and tumor necrosis factor alpha (TNF- $\alpha$ ) induce a synthesis of nitric oxide, which play an important role in the inflammation of cartilage resulting in pain. For a better understanding of the disease, animal models are powerful research tools to study the pathophysiology of diseases and the effectiveness of possible therapies. These tools contribute not only to the development of knowledge, but also to promote treatment in the medical clinic. The most relevant points in the use of animals for the study of articular cartilage are the physiological, genetic and anatomical similarities, notable in relation to humans. Animals such as mice, rats, rabbits, dogs, horses, pigs, cats, sheep, among others, are used, and animal models of osteoarthritis can be spontaneous, induced and non-evasive. Among these, pharmacological induction models (induction model of cartilaginous degeneration), traumatic induction model (surgical trauma that leads to cartilage degeneration) and genetically modified animals (for example, the suppression of a gene responsible for joint articulation).

Among this branch of research in animal models, they also stand out as histological evaluations of articular cartilage, which are one of the main tools to understand the behavior of cartilage. They are broad and diversified, according to estimates that address the degeneration of the joint complex [2]. In addition, the common histopathological evaluation methods have wide inter-observer variation and are few linear, therefore, there is an increasing need to standardize the histological evaluation of osteoarthritis. Therefore, the International Osteoarthritis Research Society published recommendations for a histological evaluation of osteoarthritis of different animals (rats, mice, rabbits, sheep, among others), being the first specific histological evaluation system for basic osteoarthritis research models. This evaluation is sensitive to detect the effects of different tissue for osteoarthritis and to facilitate reproducibility among researchers in the field. Therefore, despite the unprecedented and undeniable application, basic research in osteoarthritis aims to advance the establishment of new histological assessment tools, which have subsidy not only in the repertoire of previous publications, but also evolved in the specificity that a complete evaluation of the degenerating joint complex requires. 


\section{References}

1. Gerwin, N, Bendele AM, Glasson S, Carlson CS (2010) The OARSI histopathology initiative e recommendations for histological assessments of osteoarthritis in the rat. Osteoarthritis and Cartilage 18: S24-S34.
2. Glyn JS, Palmer AJR, Agricola R, Price AJ, Vincent TL, et al. (2019) Osteoarthritis. Lancet 386(9991): 376-387.

For possible submissions Click below: 